\title{
Planejamento e desenvolvimento de ações de Educação Permanente em Saúde na perspectiva do PMAQ-AB
}

\author{
Planning and development of Continuing Health Education actions in \\ the perspective of the $P M A Q-A B$
}

Thiala Maria Carneiro de Almeida', Rose Manuela Marta Santos², Daniela Márcia Neri Sampaio², Alba Benemérita Alves Vilela²

DOI: $10.1590 / 0103-110420195106$

RESUMO O artigo teve por objetivo analisar como ocorre o desenvolvimento das ações de Educação Permanente (EP) com base nos documentos de planejamento e avaliação das ações do Programa Nacional de Melhoria do Acesso e da Qualidade da Atenção Básica (PMAQ-AB). Como metodologia, realizou-se análise documental da Autoavaliação para Melhoria do Acesso e da Qualidade da Atenção Básica (AMAQ-AB), no período entre novembro de 2017 e março de 2018, preenchidos pela equipe gestora e pelas equipes de seis Unidades de Saúde da Família da Zona Urbana, referentes ao terceiro ciclo do PMAQ-AB; além de listas de frequências e/ou atas com ações de EP, Plano de Educação Permanente realizados pela gestão no ano de 2017 e Plano Municipal de Saúde 2014-2017. Os resultados evidenciaram que a EP é autoavaliada positivamente por ambas. Em relação aos demais documentos, constataram-se fragilidades no planejamento e execução dessas ações. Conclui-se que a AMAQ-AB figura como um novo dispositivo potente para análise da EP na Atenção Básica, sendo necessária maior divulgação de sua importância no âmbito da qualificação da Atenção Básica à saúde.

PALAVRAS-CHAVE Educação continuada. Avaliação em saúde. Atenção Primária à Saúde.

1 Universidade Federal da Bahia (UFBA) - Salvador (BA), Brasil.

thiala.maria@gmail.com

2 Universidade Estadual do Sudoeste da Bahia (Uesb)

- Jequié (BA), Brasil.
ABSTRACT The purpose of this article is to analyze how the development of the Continuing Education actions takes place based on the planning and evaluation documents of the actions of the National Program for Improvement for Access and Quality Improvement of Primary Care (PMAQ-AB). As a methodology, a documentary analysis of the Self-Assessment for Improving Access and Quality of Primary Care (AMAQ-AB) was carried out between November 2017 and March 2018, completed by the management team and the teams of six Family Health Units of the Urban Zone, regarding the third cycle of the PMAQ-AB. In addition to the attendance sheet and/or minutes with Continuing Education actions, Continuing Education Plan carried out by the management in 2017 and Municipal Health Plan 2014-2017. The results showed that Continuing Education is positively self-assessed by both. In relation to the other documents, there were weaknesses in the planning and performance of these actions. It is concluded that $A M A Q-A B$ is a powerful new device for the analysis of Continuing Education in primary care, and it is necessary to increase its importance in the qualification of Primary Health Care.

KEYWORDS Continuing education. Health evaluation. Primary Health Care. 


\section{Introdução}

O Sistema Único de Saúde (SUS) foi instituído com o objetivo de coordenar e integrar as ações de saúde das três esferas de governo, para atender, de maneira qualificada e resolutiva, às demandas de atenção à saúde'. Desde a sua criação, o SUS passou por transformações importantes, visando modificar o desenho e a operacionalização das políticas públicas de saúde, com destaque para a descentralização.

Nesse contexto, surge o Programa de Saúde da Família (PSF), posteriormente denominado de Estratégia Saúde da Família (ESF), que trouxe novas responsabilidades para a gestão e para os profissionais da Atenção Básica (AB), ao exigir mudanças nos processos de trabalho neste nível de atenção, com demandas de novas práticas de cuidado em saúde abrangendo ações de promoção, proteção da saúde, prevenção de agravos, diagnóstico, tratamento, reabilitação e manutenção da saúde ${ }^{2}$.

Diante de tais mudanças, percebeu-se a insuficiência dos setores de formação em atender às necessidades de qualificação profissional, de modo que valorizasse o potencial educativo do trabalho como fonte de conhecimento e local de aprendizagem significativa ${ }^{3}$. Nesse sentido, a Educação Permanente em Saúde (EPS) consta como estrutura da Política Nacional de Educação e Desenvolvimento para o SUS, introduzida em 2003 e vigente desde 2004, por meio de portarias e outros instrumentos de gestão do Ministério da Saúde (MS), que propõem articular aprendizagem e trabalho ${ }^{4}$.

A Política Nacional de Educação Permanente em Saúde (PNEPS), formulada com base em ideologias e movimentos de mudança na educação na saúde resultantes de concepções pedagógicas baseadas no Método Paulo Freire, busca transformar as práticas profissionais e a organização do trabalho a partir da incorporação do ensino ao cotidiano dos serviços ${ }^{4,5}$.

Neste artigo, considera-se como objetos de análise a Educação Permanente (EP), como política e como prática de ensino-aprendizagem, e os elementos trazidos pelo
Programa Nacional de Melhoria do Acesso e da Qualidade da Atenção Básica (PMAQ-AB). Este Programa foi lançado no ano de 2011, visando qualificar a $\mathrm{AB}$ como parte de uma ampla estratégia do MS denominada 'Saúde mais perto de você - acesso e qualidade'. Ele traz a proposta de avaliação e coordenação de ações para melhoria do padrão de qualidade assistencial nos serviços públicos de saúde por meio das equipes de $A^{6}$.

O PMAQ-AB está organizado em três fases e em um eixo estratégico transversal de desenvolvimento que se complementam e que compõem um ciclo contínuo de melhoria do acesso e da qualidade da assistência. O eixo transversal conta com os seguintes elementos: autoavaliação, monitoramento, EP, apoio institucional e cooperação horizontal' ${ }^{6}$.

Em síntese, as equipes de atenção à saúde e gestão, com a tarefa de imprimir mudanças na realidade concreta, deverão articular avaliação, planejamento, EP, gestão do trabalho, qualificação do processo de trabalho e monitoramento de resultados.

Assim, o eixo estratégico sugere que o saber e fazer em EP sejam integrados na prática concreta dos serviços de saúde, pois a mudança do modelo de atenção impõe a necessidade de qualificação dos serviços de saúde e de seus profissionais; além de mudanças no processo de trabalho em saúde, que passa a exigir de seus atores (trabalhadores, gestores e usuários) maior capacidade de intervenção e autonomia para que possam contribuir para o estabelecimento de práticas transformadoras ${ }^{6}$.

Dessa forma, considerando que a EP, como eixo transversal do PMAQ-AB, constitui-se como dispositivo para a qualificação das práticas de saúde no cotidiano dos serviços, bem como a importância desse Programa - que está em seu terceiro ciclo de desenvolvimento - para o fortalecimento dessas práticas, tornam-se relevantes estudos que investigam essa temática.

Em face dessas considerações e reflexões acerca das ações de EP no âmbito do PMAQ-AB, esta pesquisa tem como questão 
norteadora: como são avaliadas e planejadas as ações de EP no âmbito do PMAQ-AB em um município de pequeno porte? Para que este questionamento seja elucidado, o estudo tem como objetivo analisar aspectos relacionados com o planejamento e o desenvolvimento da EPS a partir de documentos municipais e de dados revelados pelo PMAQ-AB.

\section{Métodos}

Trata-se de uma pesquisa exploratória, do tipo documental, de abordagem qualitativa. A pesquisa foi realizada em um município de pequeno porte, situado no estado da Bahia. Esse município possui uma população estimada para o ano de 2017 em 38.259 habitantes7. A AB é composta por sete Unidades de Saúde da Família (USF) da Zona Urbana e duas unidades de zona rural ${ }^{8}$. Vale ressaltar que todas as unidades aderiram ao PMAQ-AB no terceiro ciclo.

Participaram do estudo, por meio do preenchimento dos documentos, a equipe gestora e a Coordenação da AB de seis USF da Zona Urbana, excluindo as unidades da zona rural pela dificuldade de acesso e uma unidade da Zona Urbana pelo fato de uma das pesquisadoras compor o quadro de funcionários.

Para coleta dos dados, inicialmente, foi solicitada à Secretaria de Saúde Municipal a autorização para a realização do estudo e acesso à Autoavaliação para Melhoria do Acesso e da Qualidade da Atenção Básica (AMAQ-AB) no período entre novembro de 2017 e março de 2018, preenchidos pela equipe gestora e pelas equipes de seis USF da Zona Urbana, referentes às respectivas autoavaliações feitas no terceiro ciclo do PMAQ-AB; além de listas de frequências e/ou atas com ações de EP, o Plano de Educação Permanente realizado pela gestão no ano de 2017 e o Plano Municipal de Saúde 2014-2017.

A AMAQ-AB é um caderno disponibilizado pelo PMAQ-AB que traz unidades de análise da EP para as equipes de $\mathrm{AB}$ e gestão separadamente e que envolve a avaliação do grau de conformidade de cada um desses grupos com os padrões referentes a cada item, em escala de pontuação de 0 a 10 . A nota é dada de acordo com a representação do sujeito/grupo sobre o nível de conformidade entre a situação avaliada e a qualidade desejada no padrão?

As unidades de análise estudadas por meio do instrumento $\mathrm{AMAQ}-\mathrm{AB}$ sobre os profissionais das equipes foram: formação complementar para o trabalho, participação de cursos de capacitação e qualificação, e utilização de dispositivos de educação e apoio matricial a distância.

Segundo as AMAQ-AB das equipes de saúde da família, a escala de classificação da pontuação conquistada por equipe na subdimensão EP e qualificação das equipes de $\mathrm{AB}$ está dividida em cinco categorias: de 0 a 5 pontos - Muito insatisfatório; 6 a 10 - Insatisfatório; 11 a 17 - Regular; 18 a 24 - Satisfatório; 25 a 30 - Muito satisfatório 6 .

Em relação à $A M A Q-A B$ da gestão, as unidades analisadas foram: implantação do Programa ou política de EP elaborado e instituído para a $\mathrm{AB}$, o estabelecimento de relação democrática e dialógica com as equipes, a oferta de apoio institucional para as equipes, estratégias de EP, o apoio matricial, troca de experiências, reuniões, articulações que visem a formação e aprendizagem e incentivo a cursos de qualificação, atualização e pós-graduação. A subdimensão de EP da AMAQ-AB da gestão possui a escala de classificação da pontuação dividida nas cinco categorias: de 0 a 11 pontos - Muito insatisfatório; 12 a 23 - Insatisfatório; 24 a 35 - Regular; 35 a 47 - Satisfatório; 48 a 60 - Muito satisfatório 6 .

No que tange às atas, frequências de atividades e planos, buscou-se identificar e analisar como as ações de EP foram implementadas e como ocorreram de acordo com as perspectivas do PMAQ-AB. Para a análise dos dados, as notas foram quantificadas e classificadas de acordo com as orientações da ferramenta AMAQ-AB. Os dados foram agrupados de acordo com os itens de cada subdimensão por equipe e suas pontuações, e discutidos 
à luz da literatura pertinente, sendo feito o ordenamento das equipes de forma aleatória para manter o anonimato dos profissionais que atuam nas unidades. Dos demais documentos, foram extraídos os trechos que se relacionavam com o tema; e, posteriormente, transcritos.

A etapa analítica seguiu o processo denominado análise documental, em que se tem o documento como objeto de investigação, com a finalidade de produzir novos conhecimentos, criar formas de compreender fenômenos e dar a conhecer a forma e profundidade de como estes têm sido desenvolvidos, permitindo uma compreensão do contexto histórico e sociocultural ${ }^{10}$.

O estudo atendeu às diretrizes da Resolução $n^{0} 466 / 12^{11}$, e foi aprovado pelo Comitê de Ética em Pesquisa (CEP) da Universidade Estadual do Sudoeste da Bahia (Uesb) sob o $n^{\circ}$ CAAE 77847417.9.0000.0055. Ainda assim, para a condução do estudo, foi solicitada Carta de Anuência da Secretaria de Saúde do município analisado, autorizando a coleta dos dados nos instrumentos preenchidos pelas equipes.

\section{Resultados e discussão}

A partir da análise documental, identificou-se que, no Plano Municipal de Saúde 2014-2017, um dos objetivos apresentados é o de intensificar as estratégias para o aprimoramento da política de gestão de pessoas, com vistas à democratização do processo de trabalho e da política de EP, enquanto ferramenta fundamental no processo de qualificação dos trabalhadores da saúde, a partir das necessidades de saúde das pessoas e das populações, da gestão setorial e do controle social em saúde8.

Para tanto, foi proposto o fortalecimento das ações do Núcleo de Educação Permanente (NEP), criado em 2012, de forma a consolidar a integração entre os serviços de saúde e as instituições de ensino, além de promover a construção do Plano Anual de Educação Permanente em Saúde ${ }^{\mathbf{8}}$. Contudo, apesar das intenções de fortalecimento do NEP, ele foi desativado em 2017.

Em relação ao Plano Anual de Educação Permanente de 2017, observou-se um cronograma de atividades propostas a serem executadas por profissionais da gestão e outros profissionais da rede ou de outras instituições, porém sem mencionar o termo PMAQ-AB dentre elas. No entanto, nas listas de frequências das atividades realizadas pelas equipes, $o$ tema é citado como pauta central de umas das reuniões e da capacitação que fomentou o uso do Telessaúde pelos profissionais da equipe.

Nesse contexto, o PMAQ-AB visa desenvolver ações de apoio à atenção à saúde e EP a partir de iniciativas, como: indicação bibliográfica, teleconsultoria e telediagnóstico, e webconferências. Por isso, disponibiliza ao trabalhador um apoio permanente ao exercício profissional12. Pesquisas que examinam o Telessaúde apontam que ele tem-se constituído uma estratégia de ensino-aprendizagem contribuindo para a formação permanente dos profissionais, o que tem influenciado positivamente na prestação de cuidado ${ }^{13}$.

Os dados obtidos mostram que, nas autoavaliações, quatro equipes classificaram suas ações de EP como satisfatória; uma equipe, como regular; e uma, como muito satisfatória (tabela 1). 
Tabela 1. Autoavaliação da Equipe de Saúde da Família segundo a subdimensão Educação Permanente e qualificação das equipes de Atenção Básica. Bahia, 2018

\begin{tabular}{|c|c|c|c|c|c|c|}
\hline \multicolumn{7}{|c|}{ Subdimensão: Educação Permanente e qualificação das equipes de Atenção Básica } \\
\hline Item & Equipe & Equipe & Equipe & Equipe & Equipe & Equipe \\
\hline & 01 & 02 & 03 & 04 & 05 & 06 \\
\hline $\begin{array}{l}\text { Os profissionais da equipe de nível superior e médio possuem } \\
\text { formação complementar que os qualifica para o trabalho na } \\
\text { Atenção Básica. }\end{array}$ & 10 & 0 & 08 & 06 & 08 & 07 \\
\hline $\begin{array}{l}\text { A equipe participa de cursos de atualização e de qualificação, } \\
\text { aplicando os conhecimentos aprimorados na melhoria do } \\
\text { trabalho e da qualificação da Atenção Básica. }\end{array}$ & 10 & 10 & 07 & 08 & 08 & 08 \\
\hline $\begin{array}{l}\text { As equipes utilizam dispositivos de educação e apoio matricial } \\
\text { a distância para a qualificação do cuidado. }\end{array}$ & 07 & 05 & 08 & 08 & 07 & 09 \\
\hline Total & 27 & 15 & 23 & 22 & 23 & 24 \\
\hline
\end{tabular}

Fonte: Elaboração própria, a partir da análise dos dados coletados na pesquisa.

De acordo com os dados encontrados nesta pesquisa, foi possível verificar que não foram mencionados os padrões muito insatisfeito e insatisfeito, corroborando os resultados de um estudo realizado com 75 equipes de saúde da família de um município de Minas Gerais, que teve como objetivo analisar a avaliação dessas equipes quanto aos itens EP e qualificação profissional para $\mathrm{AB}^{14}$. Todavia, no estado do Paraná, também foram encontradas equipes com avaliação correspondente ao padrão de qualidade regular ${ }^{15}$.

Vale ressaltar que essas autoavaliações podem estar propensas a um possível viés de informação pelo fato de o próprio profissional realizá-las e por não haver uma observação direta ${ }^{\mathbf{1 4}}$.

Os resultados deste estudo, quando analisados na perspectiva de cada item da subdimensão, apontam falhas na execução destas ações ou mesmo um desconhecimento de seu potencial no processo de qualificação das equipes de USF.

Outro achado importante a ser destacado nesta pesquisa diz respeito à avaliação positiva da formação qualificada e participação de cursos, tal como verificado por outros estudos $5,14,16,17$. Porém, a mesma equipe que autoavaliou suas ações de EP como regular considera que não possui formação qualificada para atuar na $\mathrm{AB}$.

Uma pesquisa que avaliou o desempenho da Atenção Primária à Saúde (APS) em Belo Horizonte, Minas Gerais, mostrou que a formação das equipes no tema saúde da família foi um fator fortemente associado ao desempenho da APS ${ }^{18}$. Tais qualificações contribuem para que se desenvolvam ações de saúde mais resolutivas, propiciando melhor desempenho técnico e profissional, assim como subsidia o planejamento e intervenções da equipe. Essas ações se constituem ferramentas para o desenvolvimento de novas habilidades e competências que visam à qualificação na $\mathrm{AB}^{9}$.

Em relação à participação em atividades de educação e a apoio matricial a distância, também foi encontrado, neste estudo, uma avaliação positiva, assim como no estudo de Aurélio Pinto et al. 5 , elaborado com dados da avaliação externa do PMAQ-AB. Esses autores demonstraram que a participação em atividades de Telessaúde e atividades de educação a distância têm frequência relevante no cenário brasileiro e revelou uma avaliação positiva das equipes de USF no quesito da EP, sugerindo que há associação entre EPS e AB. Essas atividades, além de aumentar a resolubilidade clínica dos profissionais da $\mathrm{AB}$, possibilita um canal de diálogo destes com outros profissionais e serviços?.

De acordo com a AMAQ-AB da equipe gestora, suas ações de EP foram autoavaliadas como muito satisfatória, sendo todos os itens 
pontuados com nota acima de 7, inclusive o item que se refere à implantação da política de EP no município e dois itens que se referem ao apoio institucional (tabela 2).

Tabela 2. Autoavaliação da equipe gestora segundo as subdimensões Implantação e implementação da Atenção Básica no município e Educação Permanente. Bahia, 2018

\begin{tabular}{|c|c|}
\hline Subdimensão: Implantação e implementação da Atenção Básica no município & \\
\hline Item & Gestão \\
\hline $\begin{array}{l}\text { A gestão municipal de saúde possui programa ou política de Educação Permanente elaborado e instituí- } \\
\text { do para a Atenção Básica. }\end{array}$ & 09 \\
\hline \multicolumn{2}{|l|}{ Subdimensão: Apoio Institucional } \\
\hline Item & Gestão \\
\hline A gestão da Atenção Básica estabelece relação democrática e dialógica com as equipes. & 10 \\
\hline A gestão da Atenção Básica oferta apoio institucional ou similar para as equipes de Atenção Básica. & 10 \\
\hline \multicolumn{2}{|l|}{ Subdimensão: Educação Permanente } \\
\hline Item & Gestão \\
\hline $\begin{array}{l}\text { As estratégias de Educação Permanente são construídas baseadas em temas e as necessidades defini- } \\
\text { das com os profissionais da Atenção Básica. }\end{array}$ & 10 \\
\hline A gestão da Atenção Básica utiliza o apoio matricial como estratégia de Educação Permanente. & 09 \\
\hline A gestão da Atenção Básica dispõe de estratégias que estimulam a troca de experiências. & 08 \\
\hline A gestão da Atenção Básica garante às equipes de saúde momentos destinados às reuniões. & 09 \\
\hline $\begin{array}{l}\text { A gestão da Atenção Básica faz articulações de forma a propiciar que as unidades de Atenção Básica } \\
\text { sejam espaços de formação e aprendizagem. }\end{array}$ & 08 \\
\hline $\begin{array}{l}\text { A gestão da Atenção Básica viabiliza a participação para os profissionais de saúde em cursos de atuali- } \\
\text { zação, de qualificação e de pós-graduação. }\end{array}$ & 09 \\
\hline Total & 53 \\
\hline
\end{tabular}

As notas desses itens foram superiores às atribuídas por 26 equipes de USF envolvidas em outro estudo ${ }^{19}$, fato que revela diferenças entre a avaliação realizada pelos profissionais de saúde e pela equipe gestora, podendo a primeira ser mais fidedigna, já que os profissionais podem avaliar melhor as ações que foram realizadas do que os próprios gestores.

Para o item que avalia se as estratégias de EP contemplam as necessidades dos profissionais da $\mathrm{AB}$, a equipe gestora atribuiu a nota máxima, ou seja, o valor 10 . No entanto, foi percebida a ausência de ações de EP voltadas para o PMAQ-AB no Plano Anual de Educação Permanente no período em que o município se preparava para avaliação externa, o que sugere uma fragilidade dessas estratégias e um viés na informação.
Em outro estudo realizado, foi identificado o padrão relacionado com a elaboração de atividades de EP baseadas em temas e necessidades definidas com os profissionais da $\mathrm{AB}$, o qual apresentou nota média 8. Já em relação ao uso pela gestão da $\mathrm{AB}$ do apoio matricial como estratégia de EP, a nota média foi de 7. Sobre a disponibilização pela gestão da $\mathrm{AB}$ de estratégias que estimulem a troca de experiências, obteve-se nota média $6^{19}$.

Em relação ao apoio institucional, neste estudo, os dois padrões de qualidade (relação democrática e dialógica com as equipes e oferta de apoio institucional ou similar para as equipes) também foram avaliados pela equipe gestora da $\mathrm{AB}$ com nota máxima. Esse achado apresenta consonância com estudo ${ }^{12}$ feito a partir da avaliação externa, em que a maioria 
das equipes referem receber apoio institucional permanente de uma equipe da secretaria de saúde e avaliam como muito boa ou boa a contribuição desse apoiador.

Outro estudo ${ }^{19}$ apresentou nota média 7 tanto para o padrão que trata da relação democrática e dialógica da gestão com as equipes quanto ao padrão que se relaciona com a oferta pela gestão da $\mathrm{AB}$ de apoio institucional ou similar para as equipes.

Essa variação de valores pode ser atribuída ao desvirtuamento em relação ao apoio institucional oferecido pela gestão. De acordo com Rocha et al. ${ }^{19}$, apesar de as equipes mencionarem que existe apoio institucional, seu papel pode apenas ser uma nova nomenclatura para ações gerenciais feitas por supervisores, limitando-se a questões burocráticas, o que não se configura, na prática, com a função de debater e priorizar problemas, distanciando-se dos princípios dialógicos, resolutivos e potenciais da função de apoiador no âmbito do PMAQ-AB.

Outra questão apontada por resultados de outra avaliação feita pelo PMAQ-AB é acerca da atuação de outros profissionais no papel de apoiador, como a vigilância em saúde ${ }^{20}$. Considerando o acúmulo de funções, a função do apoiador institucional pode ser negligenciada para atender ao cumprimento formal das ações do PMAQ-AB, fragilizando as ações inerentes a essa função.

O apoiador institucional é uma estratégia fundamental na fase de desenvolvimento do PMAQ-AB e responsabilidade da gestão de equipes de $\mathrm{AB}$ para garantir sua implementação. Para tal, essa função compreende: mediação e estabelecimento de compromissos mútuos, promoção e ampliação da capacidade crítica dos profissionais e ativação de espaços coletivos de aprendizado ${ }^{6}$. Dessa forma, esse elemento deve aproximar-se da EP no sentido de aperfeiçoar os processos de trabalhos e a interação entre os sujeitos.

Embora os resultados destas pesquisas sugiram a existência do apoio institucional, para a avaliação de seu desempenho, seria necessário um aprofundamento na investigação dessas ações.

\section{Considerações finais}

No presente estudo, evidenciou-se a predominância de avaliação positiva das equipes e da gestão quanto à EP, além de outras subdimensões intimamente relacionadas com a EP. No entanto, constata-se uma equipe classificada no padrão de qualidade regular, sugerindo que a EP ainda não se efetivou no cotidiano dos profissionais.

Outro achado refere-se a um Plano de Educação Permanente que ainda se encontra em construção e apresenta fragilidades na programação de suas ações.

Nota-se que o eixo de desenvolvimento do PMAQ-AB, por meio de processo avaliativo, permite reflexões na equipe de saúde para a importância da EP para o trabalho em saúde. Esse achado é corroborado pelos instrumentos de planejamento.

Faz-se necessária, portanto, a articulação da autoavaliação na perspectiva da EP por meio do PMAQ-AB. Recomenda-se planejar ações de incentivo à qualificação profissional em serviço e mediante a EP para aquisição de competências e habilidades na $\mathrm{AB}$ no âmbito do PMAQ-AB.

Como limitações deste trabalho, aponta-se aqui o seu caráter de estudo locorregional, por isso, evidencia-se a necessidade de novos estudos sobre a temática com abrangência nacional.

\section{Colaboradores}

Almeida TMC (0000-0002-8383-9519)* contribuiu para coleta, análise dos dados, escrita do manuscrito. Santos RMM (0000-00017923-7518)* contribuiu para orientação metodológica e revisão do manuscrito. Sampaio DMN (0000-0002-4195-1772)* contribuiu para orientação metodológica e revisão do manuscrito. Vilela ABA (0000-0003-2110-1751)* contribuiu para orientação metodológica e temática, revisão do manuscrito.
*Orcid (Open Researcher and Contributor ID). 


\section{Referências}

1. Silva EC, Gomes MHA. Regionalização da saúde na região do Grande $\mathrm{ABC}$ : os interesses em disputa. Saude soc. [internet]. 2014 [acesso em 2018 jan 2]; 23(4):1383-1396. Disponível em: http://www. scielo.br/scielo.php?script=sci_arttext\&pid=S0104$-12902014000401383 \& \operatorname{lng}=$ en.

2. Assis F, Mischiati MF. Retrospectiva histórica da implantação do PSF até sua transformação em ESF nos dias de hoje. Rev. Uninga [internet]. 2010 [acesso em 2019 fev 26]; 3(1):23-31. Disponível em: http:// revista.uninga.br/index.php/uningareviews/article/ view/502.

3. Ceccim RB. Educação permanente em saúde: descentralização e disseminação de capacidade pedagógica na saúde. Ciênc. Saúde Colet. [internet]. 2005 [acesso em 2019 fev 26]; 10(4):975-86. Disponível em: http:// dx.doi.org/10.1590/S1413-81232005000400020.

4. Brasil. Ministério da Saúde. Gabinete do Ministro. Portaria ${ }^{0} 1.996$, de 20 de agosto de 2007. Dispõe sobre as diretrizes para a implementação da Política Nacional de Educação Permanente em Saúde e dá outras providências. Diário Oficial da União. 21 Ago 2007.

5. Pinto AH, Ferla AA, Ceccim RB, et al. Atenção Básica e Educação Permanente em Saúde: cenário apontado pelo Programa Nacional de Melhoria do Acesso e da Qualidade da Atenção Básica (PMAQAB). Divulg. saúde debate [internet]. 2014 [acesso em 2019 fev 26]; 51:145-60. Disponível em: http:// cebes.org.br/site/wp-content/uploads/2014/12/ Divulgacao-51.pdf.

6. Brasil. Ministério da Saúde. Programa Nacional de Melhoria do Acesso e da Qualidade da Atenção Básica (PMAQ) - Manual Instrutivo $3^{\circ}$ Ciclo (2015 - 2016). Brasília, DF: MS; 2017.

7. Instituto Brasileiro de Geografia e Estatística. [acesso em 2019 ago 7]. Disponível: https://cidades.ibge.gov. br/brasil/ba/amargosa/panorama.
8. Bahia. Prefeitura Municipal de Amargosa. Plano Municipal 2014-2017. Amargosa: Secretaria Municipal de Saúde; 2014.

9. Brasil. Ministério da Saúde. Autoavaliação para a Melhoria do Acesso e da Qualidade da Atenção Básica. Brasília, DF: MS; 2016.

10. Sá-Silva JR, Almeida CD, Guindani JF. Pesquisa documental: pistas teóricas e metodológicas. RBHCS. [internet]. 2009 [acesso em 2019 fev 26]; 1(1):1-15. Disponível em: https://www.rbhcs.com/rbhcs/ article/view/6/pdf.

11. Brasil. Conselho Nacional de Saúde. Resolução n o 466, de 12 de dezembro de 2012. incorpora, sob a ótica do indivíduo e das coletividades, referenciais da bioética, tais como, autonomia, não maleficência, beneficência, justiça e equidade, dentre outros, e visa a assegurar os direitos e deveres que dizem respeito aos participantes da pesquisa, à comunidade científica e ao Estado. Brasília, DF: Diário Oficial da União. 12 Dez 2012.

12. Pinto HÁ, Ferla AA, Florêncio ARO. Programa Nacional de Melhoria do Acesso e da Qualidade da Atenção Básica; várias faces de uma política inovadora. Divulg. saúde debate [internet]. 2014 [acesso em 2019 fev 26]; 51:358-372. Disponível em: http:// www.scielo.br/pdf/sdeb/v38nspe/0103-1104-sdeb38-spe-0358.pdf.

13. Godoy SCB, Guimarães EMP, Assis DSS. Avaliação da capacitação dos enfermeiros em unidades básicas de saúde por meio da telenfermagem. R. Esc. Anna Nery. [internet]. 2014 [acesso em 2019 fev 26]; 18:148155. Disponível em: http://www.scielo.br/pdf/ean/ v18nl/1414-8145-ean-18-01-0148.pdf.

14. Moreira S, Lima CA, Vieira MA, et al. Educação Permanente e Qualificação Profissional para Atenção Básica. Saúde e Pesquisa. [internet]. 2017 [acesso em 2019 fev 26]; 10(1):101-109. Disponível em: http:// periodicos.unicesumar.edu.br/index.php/saudpesq/ article/view/5682. 
15. Rizzotto MLF, Gil CRR, Carvalho M, et al. Força de trabalho e gestão do trabalho em saúde: revelações da Avaliação Externa do Programa Nacional de Melhoria do Acesso e da Qualidade da Atenção Básica no Paraná. Saúde debate [internet]. 2017 [acesso em 2019 fev 26]; 38(esp):237-251. Disponível em: http:// www.scielo.br/pdf/sdeb/v38nspe/0103-1104-sdeb38-spe-0237.pdf.

16. Fonseca S, Machado ATGM, Lima AMLD, et al. Compreendendo o apoio matricial e o resultado da certificação de qualidade nas áreas de atenção à criança, mulher, diabetes/hipertensão e saúde mental. Saúde debate [internet]. 2014 [acesso em 2019 fev 26]; 38(esp):83-93. Disponível em: http://www.scielo.br/ pdf/sdeb/v38nspe/0103-1104-sdeb-38-spe-0083.pdf.

17. Seidl H, Vieira SP, Fausto MCR, et al. Gestão do trabalho na Atenção Básica em Saúde: uma análise a partir da perspectiva das equipes participantes do PMAQ-AB. Saúde debate. [internet]. 2014 [acesso em 2019 fev 26]; 38(espl):94-108. Disponível em: http:// www.scielo.br/pdf/sdeb/v38nspe/0103-1104-sdeb38-spe-0094.pdf.
18. Turci MA, Lima-Costa MF, Macinko J. Influência de fatores estruturais e organizacionais no desempenho da atenção primária à saúde em Belo Horizonte, Minas Gerais, Brasil, na avaliação de gestores e enfermeiros. Cad. Saúde Pública [internet]. 2015 [acesso em 2019 jun 28]; 31(9):941-952. Disponível em: http://www. scielo.br/pdf/csp/v31n9/0102-311X-csp-31-9-1941. pdf.

19. Rocha ES, Souza EC, Lima MV, et al. Perspectivas avaliativas da gestão da Atenção Básica em município de médio porte. Sanare [internet]. 2016 [acesso em 2019 ago 7]; 15(1):8-14. Disponível em: https://sanare. emnuvens.com.br/sanare/article/view/920/550.

20. Silva LAA, Soder RM, Petry L, et al. Educação permanente em saúde na atenção básica: percepção dos gestores municipais de saúde. Revista Gaúcha de Enfermagem [internet]. 2017 [acesso em 2019 jun 28]; 38(1):e58779. Disponível em: http://www.scielo.br/pdf/rgenf/v38nl/0102-6933rgenf-1983-144720170158779.pdf .

Recebido em 11/03/2019

Aprovado em 29/07/2019

Conflito de interesses: inexistente

Suporte financeiro: não houve 